\title{
The Status Quo of Chinese Medicine Education in Higher Vocational Colleges and Practice and Application of the Training Model of "Teacher Leading Apprentice" Tutorial System
}

\author{
Kai Zhou, Ming Wang
}

Zhengzhou Shuqing Medical College, Zhengzhou 450064, Henan, China. E-mail: zhoukai0202@126.com

Abstract: "Teacher succession" has always been a traditional way of inheritance of Chinese medicine. Through the succession of teachers, the valuable experience accumulated by previous generations of doctors in long-term clinical practice can be passed on from generation to generation. The advantage of this inheritance model is that it can carry out practice to the greatest extent, learn theory in practice, and better combine theory with practice, which is more in line with the characteristics of Chinese medicine education.

Keywords: Higher Vocational Colleges; Chinese Medicine; Teacher and Apprentice

In the modern education system, the common educational form for TCM majors at the higher vocational and technical level is a combination of large-class intensive theoretical teaching and a small amount of practical teaching. Compared with the traditional TCM education system, this teaching method has its advantages: more educate a group of Chinese medicine practitioners quickly to reduce education costs. However, there are also obvious problems of uneven education quality, more emphasis on theory, and insufficient practical experience. The "teacher and apprentice" education model has many influences on the training of TCM talents, from learning methods, professional knowledge, the combination of theory and practice, the establishment of dialectical thinking, the training of personal skills, and the improvement of their own quality. Students are trained in all aspects so that students can become more talented. How to achieve a balance between the two, that is, to educate more talents with higher efficiency, and to minimize the shortage, is a problem that modern Chinese medicine educators have been paying attention to.

\section{Current status of TCM education at specialist level}

\subsection{Insufficiency of TCM education mechanism}

There have always been too many contradictions between the three-year education system and the broad and profound content of Chinese medicine. One is the lack of education time leading to the lack of Chinese medicine thinking. In the teaching practice of modern Chinese medicine, the teaching time for the content of traditional Chinese medicine classics is getting shorter and shorter, which makes the Chinese medicine thinking of Chinese medicine students worse and worse, and even some students are still hesitating to believe whether Chinese medicine is scientific when they graduate. Second, the proportion of special Chinese medicine education in the entire education system is seriously insufficient, and a large amount of teaching time is spent on learning Western medicine, politics, language and other subjects, resulting in insufficient mastery of the theory of Chinese medicine, and it is even more difficult to apply it in clinical practice. Third, the short teaching time

Copyright(C) 2020 Kai Zhou et al.

doi: 10.18686/ahe.v4i10.2955

This is an open-access article distributed under the terms of the Creative Commons Attribution Non-Commercial License (http://creativecommons. org/licenses/by-nc/4.0/), which permits unrestricted non-commercial use, distribution, and reproduction in any medium, provided the original work is properly cited. 
has caused the junior college freshmen to basically have no time to carry out professional education after they enrolled. From the high school or technical secondary school professional cultural knowledge learning to professional learning, the content of Chinese medicine is even more profound. The resulting ancient and modern Chinese and foreign cultural shock and vibration, and the parallel of language and writing, can easily make students at a loss what to do.

\subsection{Problems with the concept of Chinese medicine education}

There are also many problems in the concept of Chinese medicine education. One is that as an educator of the Chinese medicine profession, he should implement the concept of Chinese medicine in his teaching and take traditional Chinese culture as the foundation. However, in the current Chinese medicine education, students and some teachers are more focused on western medicine, thinking that it is necessary to learn Chinese and Western medicine at the same time. The result is that the higher the education level, the deeper the influence of western medicine. Some graduate students even try to use western medicine to verify the experiment. The scientific nature of Chinese medicine can only result in that the clinical thinking of Chinese medicine is becoming less and less important, and students majoring in Chinese medicine gradually lose their professional confidence in this state. Second, the different cultural backgrounds of Chinese and Western medicine have led to the inability to integrate Chinese and Western medicine theories. If students are blindly required to learn Chinese and Western medicine at the same time in teaching, it will only increase the difficulty of students' learning. In the learning process, students will be confused in the two different dimensions of learning due to the different standards, and will lose the clinical thinking of Chinese medicine in the future work. Third, Chinese medicine itself is a practical science. To learn Chinese medicine, you must gradually master the skills in practice. However, the existing Chinese medicine education has too little practice time, and there are fewer teachers who can guide students in practice, and there are fewer teachers who have time and are willing to guide students seriously. As a result, students have very insufficient practical experience, and their professional confidence in clinical practice is even worse.

\subsection{Poor learning effect of students}

Students majoring in Chinese medicine in higher vocational colleges generally have poor learning effects. The reason for this problem is not only the two system or ideological problems stated above, but more problems exist in the students themselves. One is that the quality of students in higher vocational colleges is generally not high, and the students have not laid a good foundation of cultural knowledge in the high school or technical secondary school era, which is extremely disadvantageous for the study of Chinese medicine. Traditional Chinese medicine is produced on the basis of profound Chinese traditional culture. The lack of cultural knowledge can easily lead to the inability to understand the classics of Chinese medicine when learning Chinese medicine. There is a clear gap between the lack of solid foundation and the deep accumulation required to learn Chinese medicine. The second is that students' beliefs in Chinese medicine will be affected by all aspects, such as teachers' influence, teaching influence, social influence, working environment influence, etc. These influences will be concentrated on the students' unsteadiness in the Chinese medicine profession. The specific performance is that the performance of the Chinese medicine course is not satisfactory and the learning effect is not good. The third is that the learning of Western medicine is relatively intuitive, does not require cultural accumulation, and the difficulty of learning is relatively low. Students are more willing to learn relatively simple content, resulting in poor students' performance in Chinese medicine.

\section{The current status of the application of the "teacher and apprentice" tutorial system in Chinese medicine education}

\subsection{The characteristics of the teaching method of "teacher and apprentice" tutorial system}

"Teacher-to-apprentice" tutorial system teaching is the ancient teacher-to-education. Teacher education is a traditional Chinese medicine education method with the main form of the master's family history, with the main features of clinical practice, oral teaching, close cooperation with theory and practice, and emphasis on clinical practice ${ }^{[1]}$. "Teacher leads apprentice" tutor system teaching emphasizes that the tutor leads the apprentice to learn. Students carry out theoretical and practical learning under the guidance of their instructors. This method strengthens the cultivation of practical skills in Chinese medicine teaching, and emphasizes the cultivation of clinical abilities. At the same time, one-to-one teaching is also conducive to adjusting the teaching content and teaching progress according to the students' situation, but also conducive to subtle medical ethics education between teachers and students. 


\subsection{The influence of the "teacher and apprentice" tutorial system on the teaching of Chinese medicine}

The "teacher-to-apprentice" tutor system itself is the mainstream method of traditional Chinese medicine teaching, and its influence on Chinese medicine teaching is very obvious. As a practical medicine, Chinese medicine requires full practice. The tutor system teaching method is that students carry out theoretical and practical learning under the guidance and guidance of their tutors. Students master theory from practice and use theory to better guide practice. Under the guidance and help of the instructor, students can carry out clinical practice, experience the theoretical knowledge they have learned from the clinic, and learn from the experience of the instructor in practice, avoiding learning detours and reducing the difficulty and time of learning. Students can also make better use of the theoretical knowledge they have learned in practice to combine theory with practice. Carrying out your own clinical practice under the guidance of your mentor can also avoid clinical disputes. No matter from which aspect, the tutorial system is one of the more suitable methods for teaching Chinese medicine practice. However, the inability of the tutorial system to train a large number of talents at one time is also one of its problems. The efficiency is not proportional to the quality, and it cannot be widely promoted. This is also one of the challenges to the current teaching of Chinese medicine.

\subsection{Application and practice of the "teacher-to-apprentice" tutorial system}

The main inheritance method of traditional Chinese medicine from ancient times to the present is from teacher to teacher, which is the modern "teacher and apprentice" tutor system teaching. The main problems of modern teaching methods of Chinese medicine can be made up by the tutorial system, and the shortcomings of the tutorial system can be supplemented by modern teaching methods. The two complement each other and can better improve the teaching effect. At present, many Chinese medicine hospitals and schools have carried out attempts to reform the teaching of Chinese medicine through the "teacher-to-apprentice" tutorial system, and there are also many reports. For example, Henan University of Traditional Chinese Medicine has set up "Zhongjing class" in the school. At the end of the first academic year, some students are selected from the first-year freshmen to enter the Zhongjing class by way of voluntary examination. The students who enter the Zhongjing class have implemented the dual class teacher system, tutorial system classroom teaching, and set up a Chinese medicine expert database, etc., and have achieved good teaching results, and the rate of passing the postgraduate entrance examination and the employment rate is extremely high ${ }^{[2]}$. Another example is that in the clinical practice of traditional Chinese medicine, some people have adopted the teaching method of "teacher and apprentice". The survey results show that Chinese medicine practitioners trained in the form of teacher and apprentice can meet the needs of clinical Chinese medicine work and have certain aspects of clinical work. There are also reports of applying the tutorial system to nursing teaching. The survey results show that the tutorial system can improve students' interest in learning and learning effects. Some people have also tried to introduce the "teacher and apprentice" tutor system into the training of young teachers. The research results show that the traditional "teacher and apprentice" education model has played a key role in the training of young teachers in modern universities, and can improve the teaching and research of young teachers. Some people in Gansu College of Traditional Chinese Medicine also tried to adopt the "teacher leading apprentice" tutorial system in the acupuncture and massage majors, and achieved good teaching results.

\section{Conclusion}

In summary, higher vocational education is mainly aimed at employment. The "teacher and apprentice" education model of Chinese medicine can focus on learning methods, professional knowledge, the combination of theory and practice, the establishment of dialectical thinking, the cultivation of personal skills, and the improvement of self-quality to train students in many aspects, so that they can become more talented. For college-level students, teacher-to-education is also a useful attempt.

\section{References}

1. Zheng B, He X. Retrospect and prospect of the education of Chinese medicine practitioners. China Higher Medical Education 1993; (5): 19-20.

2. Xu L. Experience of the "teacher leading apprentice" mode in traditional Chinese medicine inheritance education. Journal of Traditional Chinese Medicine Management 2015; 23(4): 26-27.

3. Li Y. Investigation and analysis of the clinical ability and job adaptability of TCM physicians with apprenticeship. Journal of Chengdu University of Traditional Chinese Medicine 2010; 33(3): 92-93. 